\title{
Coming to Terms with Medical Terms - Exploring Insights from Native and Non-native English Speakers in Patient-physician Communication
}

\begin{abstract}
Using medical terminology involves a large risk of miscommunication in English-medium consultations as patients often do not know or misunderstand the terms commonly used by physicians, or even misuse terms in their interactions with medical professionals (Hadlow/Pitts 1991; Street 2003). Patients and physicians also frequently associate different meanings with the same medical terms, which further threatens patient-physician communication (Hadlow/Pitts 1991). To date most investigations on the impact of medical terminology have focused on native English speaking (NES) individuals in monolingual encounters, while insights from non-native English speaking (NNES) physicians and patients have been largely neglected. Through semi-structured interviews, this qualitative explorative study investigates the experiences of patients and physicians from diverse linguistic backgrounds in medical encounters within Australia. A particular focus is given to the way NES and NNES participants perceive and judge the impact of the meaning of medical terms on patient-physician communication. Findings suggest that both the use and meaning of medical terminology are perceived and judged very differently by individuals who come from different language backgrounds and who hold varying degrees of medical knowledge. Findings indicate that common blanket recommendations urging medical professionals to avoid or explain jargon may be futile since physicians and patient also diverge in their understanding of what constitutes jargon or medical terminology.
\end{abstract}

\section{Introduction}

Medical terminology poses one of the greatest challenges for successful patient-physician communication given that the majority of patients possess only little medical knowledge and are therefore greatly limited in their understanding of medical terms (e.g. Hadlow/ Pitts 1991; KochWeser et al. 2009; McKinlay 1975). Poor understanding, in turn, can negatively impact on patient autonomy, satisfaction and compliance and patient-physician communication in general (Fields et al. 2008; Hadlow/Pitts 1991; Street 2003). Even though examinations of patients' knowledge of medical terminology have encompassed a wide range of medical specialties such as paediatrics (Gittelman et al. 2004) or anesthesiology (Fields et al. 2008), the predominant focus of these investigations has been on NES patients. Most multicultural countries, such as Australia, are home to large numbers of NNES patients and physicians, and, consequently, medical interactions that involve NNES interlocutors are becoming increasingly more frequent in these countries. Nevertheless, there appears to be a general preference in medical research to concentrate on NES patients (Glickman et al. 2011; Murray/Buller 2007); and to a certain degree this inclination also seems to extend to research on medical communication (see Knox/Britt 2002). However, such restrictions are to the detriment of our knowledge about the problems of NNES patients (who have often been neglected), and greatly limit our understanding of how medical interactions play out in these multicultural societies, and, subsequently, if and how they differ from NES medical communication. As a consequence, our ability to make well-informed suggestions that could help to ameliorate communication difficulties in 'cross-linguistic' consultations is also restricted.

\footnotetext{
* Maria Dahm

Department of Linguistics

Macquarie University

North Ryde NSW 2109

Australia

maria.dahm@mq.edu.au
} 
Even though the number of investigations on cross-linguistic medical interaction has increased during the past decades (e.g. Frank 2000; Pauwels 1994; Pilotto et al. 2007; Roberts et al. 2005), only a few scholars have addressed the impact of medical terminology on English-medium consultations involving NNES patients (Cooke et al. 2000; Levin 2006). As far as NNES international medical graduates (IMGs) are concerned, potential difficulties regarding the use of medical terminology, such as an overreliance on the use of medical English that can make them appear uncaring, have been, with a few exceptions, (e.g. Bates/Andrews 2001) mostly mentioned in passing, while other linguistic challenges such as pronunciation or vocabulary difficulties have been documented in greater detail (e.g. Pilotto et. al 2007).

What is more, all of these studies investigating medical terminology and its impact on consultations have been based on the premise that the concept of medical terminology can be universally defined and that interlocutors share the same concept of a 'medical term', regardless of their medical or native language background. However, research has yet to explore whether patients and physicians with different language and medical knowledge backgrounds align or diverge in their perceptions of what constitutes 'medical terminology'. Further research could also shed some light on what attitudes NES and NNES patients and physicians hold towards medical terminology, and investigate what impact the use of medical terms can have on cross-linguistic and/or cross-experiential medical communication.

The present qualitative study employs semi-structured interviews to explore how NES and NNES patients and physicians from various language backgrounds perceive and feel about the use of medical terms in patient-physician communication. By gathering individual perspectives regarding medical terms, this study also addresses the gap in the research on the impact of terminology in cross-linguistic medical communication. It is hoped that the findings can help to inform a multitude of training programs ranging from (intercultural) communication courses for medical professionals, to health literacy classes for patients, or even more specific units in programs such as the Australian Adult Migrant English Program (AMEP) that target migrants' English language needs with regard to accessing and utilising medical services in English-speaking countries.

\section{Background}

\subsection{Growing numbers of NNES patients and international medical graduates (IMGs) in Australia}

In 2009-10, net overseas immigration contributed to more than half (57\%) of the overall population growth in Australia, with more than a quarter (27\%) of the Australian population having been born overseas (Australian Bureau of Statistics (ABS) 2011). The latest Australian census data show that about 3.2 million people in Australia do not speak English at home, and just over half a million people report that they do not speak English at all or not very well (ABS 2008). It has been shown that, unless these individuals have access to and make use of professionally trained interpreters, they can encounter great communication struggles when trying to access and benefit from medical assistance in Australia (Allotey/Reidpath 1999).

The Australian population is constantly growing and also growing older, and considerable strain is put on the medical staff all over the country. For some decades now, but with increasing efforts since the 2000s, large numbers of IMGs have been recruited to counter the medical workforce shortage (Birrell/Schwartz, 2006). Although traditionally many IMGs migrated from the UK, nowadays the majority of IMGs (55\%) come from nations such as Sri Lanka or China where English is not an official language (Birrell/Schwartz 2006). Often speaking English as a second or foreign language only, IMGs are reported to encounter various linguistic difficulties in interactions with both patients and colleagues (e.g. Pilotto et al. 2007). 


\subsubsection{Medical terminology and technicalness}

Most studies investigating the effects of medical terminology on patient-physician communication conclude that the majority of patients lack proper understanding of medical terms and as a consequence frequently misuse or misinterpret them (Hadlow/Pitts 1991; Koch-Weser et al. 2009). Moreover, many patients are unaware that they misuse terms and do not realise that many common medical terms may diverge in meaning when they are used by physicians as opposed to when they are used by patients (Hadlow/Pitts 1991).

In order to prevent miscommunication, medical communication textbooks frequently urge medical professionals to avoid or adequately explain jargon (see e.g. Groves/Fitzgerald, 2010; Silverman et al 2005; Washer 2009). Generally, the term 'jargon' simply refers to technical vocabulary required to allow quick information transfer between specialists (see Washer 2009); but when used in discussions of someone's communication skills, 'jargon' usually carries more negative connotations, i.e. the unnecessary use of technical terminology in situations where it is likely that terms will be unintelligible to the other party involved. Physicians and patients, however, differ in their understanding of which vocabulary items constitute medical terms (Bourhis et al. 1989). It might, therefore, prove equally problematic for medical professionals to decide which medical terms are, indeed, jargon and should be avoided.

A range of theoretical lenses can be utilized to describe and analyse these mismatches in the perception and understanding of technical language. For example, Tannen/Wallat (1987) focussed on diverging knowledge schemas; other scholars such as Read (2004) or Schoonen/Verhallen (2008) drew on the idea of varying levels of deep word knowledge in their investigations of second language vocabulary acquisition; and within the same field, Chung (2003) and Nation (2001) relied on the concept of technicalness which describes the flexible nature and shifting meaning of (semi-technical) medical terminology. However, while all of these are very important concepts in discussing the impact of technical terminology used in patient-physician communication, to stay within the overall scope of the paper, I would like to concentrate on the idea that mismatches in the perception and understanding of technical language are caused by differences in the perception of technicalness.

The concept of a continuum of technicalness was introduced by Nation (2001) and Chung (2003) and refers to the strength and flexibility of a term's connection to its field of specialty. For example, the term migraine is closely connected to the field of medicine. The closer and more inflexible the connection of a term is to its field, the more technical the term will be. For instance, migraine is relatively flexible in its connection to the medical realm, and, in comparison to terms such as progeria (a genetic condition causing symptoms of premature ageing), migraine can be used quite easily in everyday as well as medical discourse while, progeria is confined to the latter. Following from this, I suggest that the technicalness of some medical terms is not fixed but fluctuates, because many medical terms can be used by physicians within the field of medicine, but also by patients in everyday discourse. Consequently, shifting technicalness may be one of the causes for the meaning divergences identified by Hadlow/Pitts (1991). It also seems likely that both medical background knowledge and native language status may influence how individuals perceive the technicalness of a term and, in turn, which meaning they associate with it. Yet to date, the connections between perceived technicalness, meaning divergence, medical knowledge and language background have not been tested.

\subsubsection{Medical communication in linguistically diverse communities}

Research focusing on NNES individuals in English-medium consultations has documented differences in intercultural medical communication and used these insights to develop cultural competence programs for medical professionals (see e.g. Helman 2007; Perez/Luquis 2008). As far as the role of medical terminology is concerned, investigations have been predominantly centred on monolingual English encounters (e.g. Fields et al. 2008; Gittelman et al 2004; Schouten/Meeuwesen 2006). Only a few researchers have attempted to illuminate the influence of medical terms 
on cross-linguistic medical encounters and they have discovered that, just as in monolingual interactions, comprehension difficulties also put great strain on communication between NNES patients and NES health professionals (Cooke et al. 2000; Frank 2000; Levin 2006; Pauwels 1994).

NNES IMGs working in NES medical environments in Australia have been faced with significant linguistic and communicative barriers (McDonnell/Usherwood 2008; Pilotto et al. 2007). Due to their limited English proficiency, many IMGs have problems picking up on their patients' verbal cues or efficiently communicating medical information to them using lay-friendly language (e.g. Pilotto et al. 2007; Dahm 2011).

\section{Research Aims}

The main goal of this study is to determine how participants from diverse native language backgrounds and with different levels of medical background knowledge experience the use of medical terms in consultations in Australia. To meet this objective the investigation aims to answer the following questions and in doing so maintains a distinction between participants who are a) trained physicians and patients or $\mathrm{b}$ ) from non-English speaking backgrounds in comparison to those who are NES.

1. Attitudes towards and beliefs about medical terminology.

- Which attitudes and beliefs are expressed regarding the use and understanding of medical terminology among participants?

2. Awareness of meaning divergences and influence on perceived technicalness.

- Which levels of awareness are expressed in relation to meaning divergences of medical terms among participants? How does the recognition of meaning divergences (or lack thereof) influence the way in which participants perceive the technicalness of a medical term?

3. Impact on medical communication.

- What impact does native language have on attitudes, beliefs, level of awareness and differences in perceived technicalness, and what are the consequences and implications for medical encounters involving NNES patients or IMGs?

\section{Methods}

\subsection{Procedure and participants}

For this exploratory study, semi-structured interviews were designed to generate qualitative insights into medical communication in Australia as experienced by NES and NNES patients and physicians. A special emphasis was given to perspectives on and perceptions of medical terminology as used in the Australian context. NES and NNES patient participants $(n=17)$ were drawn from a convenience sample of undergraduate students enrolled in an introductory linguistics course at an Australian University. Medical professionals $(n=11)$ were recruited with the assistance of contacts at the Royal Australian College of General Practitioners (RACGP) and the Australian School of Advanced Medicine, and included eight NNES IMGs and three NES Australian trained physicians ( 2 surgeons, 1 general practitioner). Table 1 provides a demographic overview of all participants. 


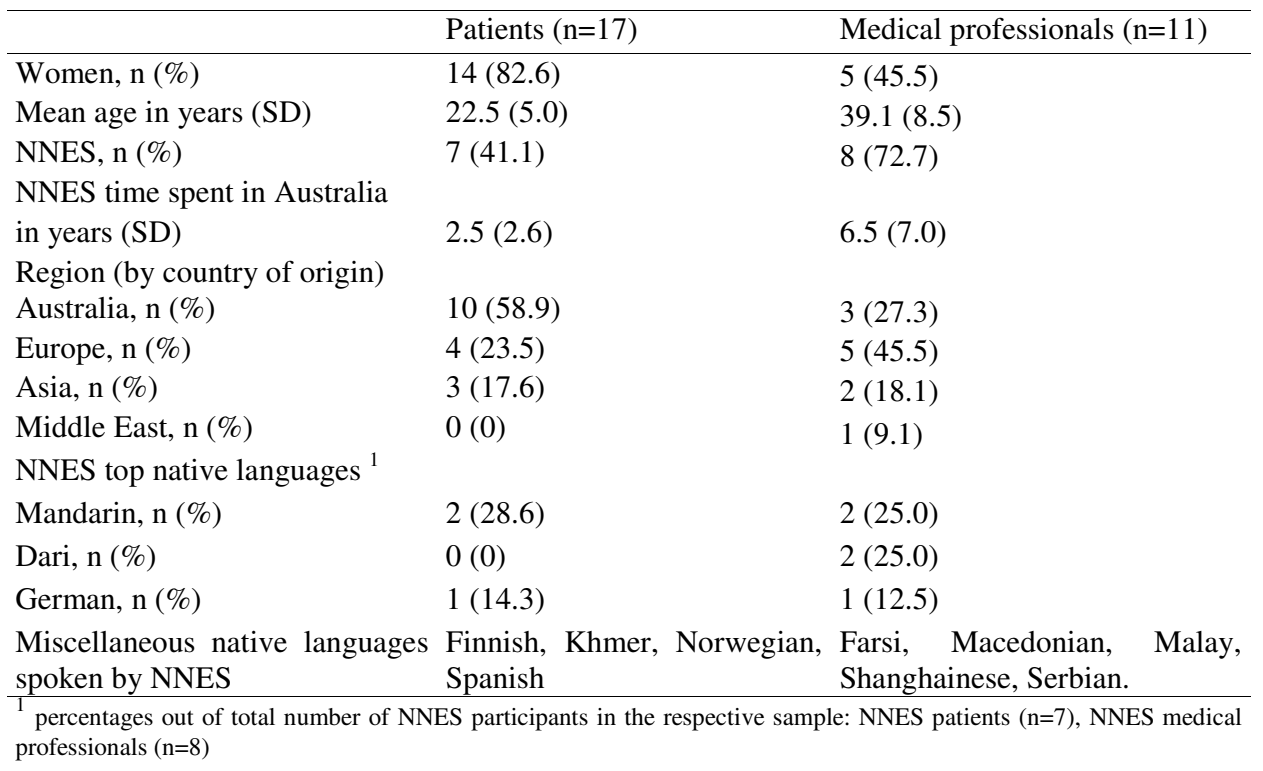

Table 1. Participant demographics $(\mathrm{n}=28)$

The set of questions in the interviews varied slightly for patients and medical professionals to account for varying levels of medical background knowledge and the different expectations they might associate with medical consultations. Additional questions for NNES participants allowed them to compare experiences gained in the Australian medical context to those of their home countries. An initial draft of the interview questions was examined for medical and research appropriateness by a lecturer in applied linguistics at an Australian university who holds degrees in both medicine and linguistics. Based on the lecturer's feedback, some questions were omitted, while others were extended or reworded. Additionally, pilot interviews were conducted with one NNES and one NES volunteer and resulted in further minor wording adjustments. These trial runs also highlighted the importance of being vigilant in the clarification of vague responses, and to continue probing in order to saturate each topic. Following one volunteer's feedback, participants were actively encouraged to share anecdotes and experiences in an effort to prevent the interviews from becoming too interrogative (see Appendix A for interview schedules).

All interviews were conducted by the author and held in English, either in the privacy of meeting rooms at an Australian university, the RACGP offices in North Sydney, or in the practices of participating Australian physicians in the Sydney metropolitan area. To avoid participants feeling coerced into disclosing intimate or confidential medical details, they were reminded throughout the interview process that they did not have to divulge any information they felt uneasy discussing. The study was approved by the Human Ethics Committee of an Australian university and approval was given by the RACGP to recruit participants and conduct interviews on their premises.

\subsection{Analysis}

All interviews were audio-recorded and de-identified; in the Findings section individual participants are referred to using pseudonyms. All analysis was conducted by the author and followed a reiterative approach (Richards 2003). Preliminary analysis started immediately after each interview ended, and consisted of briefly summarising the interview and collecting initial reflective thoughts and comments on a separate cover sheet. Subsequently, all interviews were transcribed for further analysis and ultimately coded in NVivo 8 (QSR International Pty Ltd 2008). Three aims were pursued in coding the interview transcripts: 
1. to reveal participants' attitudes and insights regarding the use of medical terminology in English-medium medical consultations in Australia;

2. to explore linguistically relevant arguments expressed by participants in relation to the research questions (see Section 3);

3. to uncover how the participants' medical background knowledge and native language status can influence beliefs about and behaviour in medical communication in Australia.

Data were coded reiteratively and coding progressed through three stages. Initially, labels were assigned freely to the transcripts using a line-by-line approach (Richards 2003). The next step in the analysis used those preliminary labels to develop systematic categories, taking into account first notions of potential links between labels (Richards 2003; Strauss/Corbin 2008). The final stage involved relating categories back to the key concepts of the investigation: professional medical knowledge and native language status. The reiterative approach in coding allowed for a continuous interpretative process (Heigham/Croker 2009). Because each new coding stage was informed by insights gathered in the previous stages, stronger conceptual relations between categories were able to emerge. Ongoing reflections and comparison throughout the coding stages ensured theoretical saturation (Richards 2003).

\section{Findings}

At this point, the exploratory nature of this study needs to be emphasised; the aim is not to provide definite answers, but to suggest avenues for more detailed investigations in the future. The main findings are discussed in more detail in the following sections; Section 5.1 focuses on attitudes and beliefs expressed by participants in relation to medical terminology; Section 5.2 addresses the participants' awareness of meaning divergences and their perceptions of medical terms and Section 5.3 offers some insight into the impact of medical terms on cross-linguistic consultations. Unedited participants' quotes are used throughout to support and illustrate the preliminary findings that emerged.

\subsection{Attitudes towards and beliefs about medical terminology}

\subsubsection{Preferences for the amount of medical terms used in consultations}

Interview data indicated that participants have different ideas about the appropriate number of medical terms to be used in patient-physician communication. It seemed that neither patients nor physicians have a universally agreed preference for plain, medical or a mix of plain and medical language in medical consultations. However, it was important for many participants that physicians should adapt the amount of medical terminology to individual patients, taking into account factors such as English proficiency, type of illness, type of consultation and previous medical knowledge. The majority of NES participants (regardless of medical background) acknowledged that, depending on the particular circumstance and after all other factors have been considered, it can be beneficial to use medical terms on an individual basis. These participants also pointed out that medical terminology can facilitate quick and efficient patient-physician communication. Similar insights were voiced less often by NNES participants.

\footnotetext{
[Doctors] still should be able to use the terminology. Because I mean if you do need to look it up or if you do wanna talk to a doctor you know or that is probably is good to actually know the correct term [...]. Rebecca (NES patient)
}

On the other hand, there was a small group of patients who, regardless of language or medical background, believed that using medical terminology is not just optional for physicians, but that they need to do so in order to show their medical competence. Some of them also called attention to the fact that it is not just using large amounts of medical terminology that signals medi- 
cal competence, but the ability to gauge which terms are actually necessary and which need to be explained:

\begin{abstract}
I think I would rather have a doctor use terms and explain them than never use terms, yes. Because the using terms is kinda like "yes, he's a doctor, okay he knows what he is talking about." [laughs]. Nadine (NNES patient)
\end{abstract}

\title{
5.1.2. Perceptions of the amount of medical terminology used in consultations
}

Discrepancies between the medical professionals and patients emerged upon analysing answers to the questions asking participant to judge the amount of medical terminology used in consultations (see Appendix for questions 8 and 9 for patient and physicians, respectively). During analysis participants' answers were coded on an ordinal scale ranging from 'low' to 'just right/okay' to 'high'. Regardless of their native language background, patients thought that more medical terms were generally used in consultations than did physicians. None of the patients judged the amount of medical terminology to be 'low'. Many patients also often expressed the view that the number of terms in consultations should be reduced because the unwarranted use of large amounts of unexplained medical terminology can make physicians appear unprofessional, uncaring, or stressed:

Well that kind of doctor would be uhm...someone who is not really considering uh... the patient or uh like the level of knowledge they have. Matti (NNES patient)

Uhm I think- usually when it is busy the doctor does tend to push forward, get straight to the point of what's wrong rather than having a long conversation and explaining everything. Kate (NES patient)

No consistent pictures emerged for physicians and they were found to either consider the number of medical terms to be 'low', or aligned with the patients' perceptions, in that they rated the occurrence of terms as either 'high' or 'just right'.

In relation to beliefs about the kind of language that should be used in consultations and their perception of the number of terms that are used in everyday practice, IMGs confessed being upset with themselves if they accidentally used a medical term out of habit or were unable to find plain vocabulary in time.

I think [medical terms] are really difficult. Because sometime there is sudden blank you can't think about medical term until you try to use the other word to explain or the what's the meaning [laughs]. Clara (NNES IMG)

All physicians repeatedly stressed that in speaking to patients, medical terms should be used carefully, but IMGs seemed to be under the impression that terminology should be avoided as best as possible. Given the small sample size, it could not be ascertained whether they did so by personal choice or whether they felt obliged to use plain language in order to pass the exams required to practice in Australia:

But when I prepare for clinical examination, I became conscious about that. So I shouldn't use [terms] with the patient because the patient is uhm is an ordinary people. So then I made my mind 'no'. With the patient I have to be very very clear and very uhm... just ordinary language. Aeisha (NNES IMG)

Interpretations of these observations, however, would be highly speculative and a larger data sample would be needed to reveal the origin of their conviction and to investigate how the IMGs' attitude towards medical terms might influence cross-linguistic medical interactions.

\subsubsection{Emotional responses to medical terms used in consultations}

Regardless of linguistic and medical education background, physicians were found to largely align with the attitudes and mostly negative emotional responses expressed by patients in reaction to the use of medical terminology in consultations. Patients described that they were most troubled by the use of unfamiliar medical terms because it caused them to feel insecure, inferior and anxious. If their conditions were labelled by medical terms, patients also appeared to perceive 
them as more serious. The physicians interviewed here confirmed that in their daily practice such emotional reactions occurred frequently. Table 2 summarizes the most frequently mentioned emotional responses.

\begin{tabular}{ll}
\hline Feeling Quote & Patients would [...] probably be overwhelmed by the amount of information that is given out and just \\
Insecurity & would like not take in what [the doctor] is saying. Kate (NES patient) \\
The patient will feel very confusing about the special terms of words, because they might did not \\
understand about the word. Lily (NNES patient) \\
If you use [medical terms] and don't explain them the patient can come out completely confused. Sara \\
(NES physician) \\
A lot of people are intimidated by doctors. Mel (NES patient) \\
I didn't want to ask too much more because he seemed to be getting a bit frustrated. Rachel (NES patient) \\
Well I think to ask questions cause they are afraid. Because the doctor is up here and they're down there. \\
Dean (NES physician) \\
It might stress them because also big words can seem a bit scary at times and... they might worry that it is \\
something more serious than it is. Rebecca (NES patient) \\
Yeah probably I feel a lot of medical terms just sounds really a lot scarier when it's in medical term. Astrid \\
(NNES patient) \\
And the [doctors] used all these words that I had no idea what it was and that kind of scared me a bit. Mel \\
(NES patient)
\end{tabular}

Table 2. Common emotional responses to the use of medical terminology

Nevertheless, the overall impact of medical terms seemed to be less straightforward and varied between participants and contexts. The following two deviant cases are used to illustrate this point. Firstly, it has previously been shown that a small number of patients appeared to assign a certain level of prestige or medical competence to the use of terminology. This association of professionalism and the use of medical terms stands in contrast to the belief held by the majority of participants that the use of too many unexplained terms signals careless and insensitive physicians showing off their knowledge. Secondly Jessica, one of the NES patients, expressed contrasting emotional effects; on the one hand, she maintained that she sometimes found comfort and a feeling of safety in the use of certain familiar medical terms, but on other occasions during the interview, she also stated that unfamiliar terms could cause her to feel concerned and anxious:

\footnotetext{
If a terminology comes up that I've heard before I definitely feel more secure and comfortable with the situation. [...] I was just so frustrated because- when [the doctors] use the big term they they scare you. Jessica (NES patient)
}

This means that her views did not only deviate from those of participants who associated medical expertise and prestige with the use of medical terms, but also from those of participants who exclusively described negative emotional responses upon encountering medical terminology in consultations.

\subsubsection{Patients' native language background and understanding medical terminology}

Within the disciplines of applied linguistics and lexicography, a lot of attention has been given to the question of what it means to truly 'know' or 'understand' a word (see e.g. Nation 1990, 2001; Schmitt 2000). Unfortunately, it would be beyond the scope of this paper to discuss in greater detail the intricacies involved in the concept of 'understanding'. Nevertheless, it should be noted that vocabulary knowledge and understanding is inherently complex and requires learners to mas- 
ter various features such as meaning, written and spoken form, grammatical behavior and pragmatic functions for each language item (see Nation 1990). As a consequence, second language learners and even native speakers might have various degrees of word knowledge or 'understanding' for different words, and understanding should therefore generally be seen as fluctuating rather than fixed. Additionally, situational context is also often needed to fully interpret the meaning of a word. In relation to using terms in interactions, it can be argued that knowing and understanding certain terms might on most occasions be accomplished as the interlocutors co-constructed the meaning using the situational context as a guide (Schmitt, 2000). However, it might be especially difficult to acknowledge the need to co-construct the meaning of semi-technical medical terms in interactions as both patients and physicians might assume a common, shared core meaning where none exists.

Data indicated that native language background may advantage or disadvantage patients and influence their understanding of and beliefs about English medical terminology. When NES patients were asked whether their native language status offered them an advantage over NNES patients, they gave very cautious answers. Many pointed out that they might enjoy greater familiarity with medical terms than NNES patients, simply because they had been exposed to them more frequently. Nevertheless, most of the NES patients immediately put their advantage in perspective and highlighted that being a native speaker would not guarantee that they actually knew the meaning of every term:

I'm sure I would [have it easier in Australian consultations] only because- yeah it is in English and I
think it would be quite hard if it wasn't your first language to sometimes understand some of the terms.
But actually I think it's hard for everyone [...] those big terms you don't use in everyday English any-
way [...] and you still wouldn't really know. Rebecca (NES patient)

In contrast, many NNES patients reported that native speakers in general enjoy certain advantages. For example, Astrid felt that NES patients "probably have some problems, but they have heard the words before so it's not as difficult as for me." Because of their limited English proficiency and limited opportunities to encounter and learn English medical terms, some NNES patients felt disadvantaged in medical interactions:

We never learned before the special terms so we are not able to use it [...] But if I learn [medical terms]
so I will try to use it. Lily (NNES patient)

[B]ecause I never speak English before so... learn from heart and the medical term. I am still learning. Suri (NNES patient)

When it comes to understanding medical terms, the NNESs' specific language backgrounds also appeared to play to an individual's advantage or create a further comprehension barrier. For example, Juan, a native Spanish speaking patient, reported that he was quite comfortable with English medical terms because they were similar to words in his native language and he was able to make sense of a term by interpreting its parts. Conversely, Amy, a native speaker of Mandarin, reported that she struggled with English medical terms because in comparison with terminology in her native language, the English terms lacked transparency.

In Chinese we just made up 'lung' and 'cancer'. So it's better for us... to understand. [...] Most of the Chinese ones I can understand because I understand the parts. But English is long and I don't know parts. Amy (NNES patient)

\subsection{Awareness of meaning divergence and perceived technicalness}

\subsubsection{Experience and awareness of meaning divergence}

Overall findings indicated that participants differed in their awareness of meaning divergences. Some physicians independently identified this issue as one of the biggest challenges in medical 
communication and were quick to state that misuse of terms by patients gives much scope for miscommunication:

And there are some terms in my specialty that I hear all the time- $[\ldots]$ but which I know from experi-
ence are uhm have a different uh common usage. Dean (NES physician)

For most of the other patients and physicians this obstacle was less obvious. They either did not acknowledge the potential for divergent meanings at all or only after being asked directly.

Unfortunately, no data could be collected from four NNES patients, because they had difficulty understanding the actual question, even after it was repeatedly rephrased and examples were used to better illustrate the point. The majority of patients, when asked directly, acknowledged that individual speakers may interpret and use terms differently. Most of them, however, seemed to be only aware of potentially troublesome meaning differences because they had experienced the impact of meaning divergences themselves, or because they had been otherwise affected personally, e.g. through an illness in the family.

Because the term 'migraine' is thrown around a lot and people... some people who get headaches say they have migraines and they are totally different [...] If I get a migraine I have to call in sick for work, like I feel I need to describe what I get... because it's not just a headache. Rebecca (NES patient)

Often physicians also drew from their experiences when discussing meaning divergence. In contrast to patients, however, physicians seem to rely on their practical rather than personal experiences. Over the years they had been in contact with a range of different patients from different linguistic, cultural and social backgrounds, all of whom they claimed frequently misuse and misinterpret medical terms. As a consequence, most physicians reported that they try to avoid certain terms as best as possible because they frequently cause confusion or create miscommunication in consultations. These items include diabetes, inflammation, infection, tumour and cancer; terms that at first glance appear be relatively well known among patients.

\subsubsection{Native language background and awareness of meaning divergence}

Data suggested that the native language background of patients and medical professionals is likely to influence how and what individuals perceive to be a medical term and may subsequently be linked to the kind of language they use or seek to clarify in interactions. For instance, some of the NNES IMGs asserted that they expected fewer problems in using medical terms when communicating with NES patients, because as native speakers, they would have less difficulty understanding terminology than NNES patients. This belief was shared by some NNES patients and appeared to be based on an assumption that because medical terms are more frequent in the English language, even in everyday discourse, they would also be better known among the general public:

It's pretty much the same like in Germany that you try to explain the difficult words but in the English speaking countries more Latin words are used in the normal language. That's what I think so you can use more uh medical terms. Karl (NNES IMG)

For example, $\mathrm{Hu}$ was convinced that the term inflammation is relatively well known amongst NES patients in comparison to NNES Chinese patients:

English speaking [patients] they can diff- differentiate what is anti-inflammation drugs and what is antibiotics. But this idea in Chinese terms they are always confused. They think inflammation- all the inflammation is caused by bacteria or viral infection, and they think antibiotics is- the drug antibiotics is a drug of inflammation medicine. Hu (NNES IMG)

Eshe, an NES patient, also commented on the understanding of the term antibiotics which is commonly used in English everyday discourse, but highlighted that not all patients actually know the correct meaning:

I think [antibiotics] is a medical [term] but is has just been so overused that is has become like... what its actual purpose is compared to like- I think people just think 'oh I need an antibiotic' but it's not used for everything that people think it's used for. Eshe (NES patient) 
As previously illustrated, most NES patients actively opposed the idea that they know the meaning of medical terms just by being a native speaker. Eshe's comments further suggest that NES patients view their own capabilities more realistically. Overall, NES patients' insights appear to challenge the assumptions many NNES participants hold about the benefits inherent in being a native speaker, and illustrates that NES individuals in fact do not necessarily know and understand more technical terminology than NNES individuals.

\subsubsection{Professional medical knowledge and perceived technicalness}

From the data it also emerged that medical background knowledge may play an influential role in shaping participants' awareness of meaning divergences and perception of technicalness. Despite the fact that physicians reported that they often deliberately avoid medical terms, a large number of patients still felt that too much terminology was being used in consultations. Medical professionals, especially those of NNES background, repeatedly pointed out that they often employ medical terms in interactions with patients out of habit (as described in Section 5.1.2), but also because those terms come to them more naturally:

Uhm at first I'll- I will use the terms or terminology at first. Because this is what I think. Hu (NNES IMG)

Jessica, one of the patients who felt that the number of terms in consultations is generally very high, speculated that '[p]erhaps a lot of the doctors don't even realise that when they're speaking that their patients don't understand the terminology that they're using.' Mel, another NES patient, went even further and suggested that the time and effort medical professionals have spent to acquire their medical knowledge have also caused them to lose their sensitivity for technical concepts and terms that only medical experts are familiar with:

\footnotetext{
Maybe [doctors] just especially GPs they see so many people a day that it sort of becomes second nature to say things in the terms that they have learned them rather than try and translate them through their brains into simple words. Mel (NES patient)
}

Consequently, it appeared to be the case that patients and physicians had different perceptions regarding the appropriateness of using medical terms in consultations. Furthermore, their judgements of what actually constitutes a medical term, and hence is appropriate to use or not, can deviate because they rely on different levels of medical background knowledge when they form their concept of a technical term. It follows that, for example, Hu's initial preference to use terms might reflect the way he thinks but could also signal that, through years of medical training and practice, he (and other medical professionals) might no longer realise that, to them, certain medical terms that have become general language items. These language items, while not perceived as technical by doctors, are frequently used in interactions with patients, and may in fact, be perceived as medical terms and judged as inappropriate by patients since they lack any medical background training. Therefore, individuals who possess no professional medical knowledge might also judge the number of terms used in consultations differently than those who have studied and practiced medicine for years.

\subsection{Impact on medical communication}

\subsubsection{Consultation time and patient information-seeking behaviour}

An unexpected finding that emerged from the data was that the use of medical terms seemed to have the potential to influence patient participation and also alter participants' perceptions of consultation length (for more information see also Dahm 2012). Many medical professionals noted that consultations were significantly prolonged when they made efforts to explain or clarify the meaning of medical terms. They indicated that such explanations were necessary either when the physicians used terms and needed to ensure that their patients would not inadvertently misinter- 
pret the terms, or in cases where patients used medical terms and the professionals felt the need to clarify because the patient might associate a different or even unique meaning with the term.

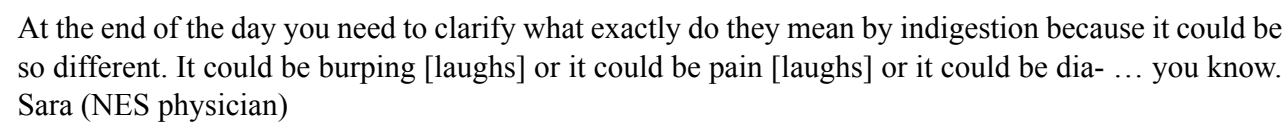

While patients stated that they do ask their physician for clarification, most patients also pointed out that they often refrain from asking their physician to explain unfamiliar terms; either because they felt they were wasting the physician's time or because they do not want to disclose any of the negative feelings mentioned in Section 5.1.3.

From the data it emerged that patients used very different strategies when actively seeking information from their physician. Some patients expressed that they may hesitate to ask for clarification because they did not want to admit they were lost, and a few individuals even spoke of actively disguising their confusion by nodding and pretending to be interested. Rather than asking their physician for further information, most often these participants, such as Deniz (NES patient), would "google [terms] to the ends of the earth" to find information that went beyond mere definitions.

But yeah I think I was a bit embarrassed at the time that's also why I didn't ask her. That's why I looked it up later on Wikipedia. Mary (NES patient)

Another group of patients, especially those from/with NNES backgrounds, were also reluctant to address the physicians, but they seemed to avoid asking questions because they viewed the professional as an unquestioned authority:

But sometime when I ask too much I'm scare....uhm... I scare people might not be happy when I ask them. [...] in Cambodia I never ask for 'can I do this, can I do that?' And we believe what the doctor do is right. Suri (NNES patient)

In contrast to the other patients, who preferred to look for more information online, some of the NNES patients also stated that they were interested first and foremost in looking up the translation of unknown lexical items and not in seeking additional information. They cited bilingual dictionaries in book or electronic (including online dictionaries) format as their sole resource for information:
Lily
Yeah I actually check for the dictionary- uhm the electronic dictionary and In ternet for dictionary as well.
Researcher
[...] Do you ever go and type a term into Google and see where it takes you?
Lily
No. Because it takes time [laughs].

\subsubsection{Using medical terminology in consultations}

As suggested in the previous sections, participants from different language and medical knowledge backgrounds held different attitudes towards and beliefs about the use of medical terms, which indicated a large risk of miscommunication. Various differences emerged among patients in relation to their experience with, and subsequent use of terminology. All patients offered predominantly negative comments on (initially) unfamiliar medical terms. After the first encounter with a medical term, however, most patients often independently sought medical background information to understand certain terms better. This increased knowledge seemed to improve their feelings towards medical terms and they stated that they would start to use terms more freely and without apprehension:

\footnotetext{
Because when I tell people I have like polycystic ovarian syndrome I use that like big term and no one knows what it is, so I always have to explain it. [...] but you know it from personal experience because you look it up and you find out about it. Mary (NNES patient)
} 
Other patients, mostly NNES patients who lamented their poor English knowledge, did not mention adopting terms into their vocabulary after researching online and stated that most of their communication was just guesswork. The lack of transparency in English medical terms that was addressed in Section 5.1.4 also further limits the ability of those patients to guess the meaning of unfamiliar terms - a strategy that $\mathrm{Hu}$ describes as being frequently used by patients in China to avoid having to ask for more information or clarification directly:

The Chinese medical terms is much easier for the patient to understand than the English medical terms. [...] Anyone- even guess- they can guess fifty percent but in English terms they- they know that they don't know it [...] Hu (NNES IMG)

Guesswork is far from an ideal solution to develop an understanding of unfamiliar medical terms and, in fact, incorrect guessing or failure to seek explanations may have serious implications. For example Suri, an NNES patient from Cambodia, tried to guess the meaning of the term appendix and did not ask her physicians to clarify it for her "because [she was] too shy to ask actually:"

First is hard to understand what they- what they say to me what my problem. Yeah and I just guessing... uhm... yeah generally guessing what they're saying [...] because I was too scared to ask... because too shy to ask actually [laughs]. [T]wo specialist caming and tell me what's happening, what they have to do but there's a uhm... small.. uh word that I can't understand. They use a lot of terminology word and that... I have no ideas [and]- I didn't ask. Suri (NNES patient)

Furthermore, due to her level of English, it seems likely that Suri often encountered unfamiliar English words, and may not even been able to identify this particular unknown word as a medical term:

Uhhmm.. Appendix. First time I heard the word I don't think is a medical term [...] Just a word to me... just a word I never heard. Suri (NNES patient)

Suri only really understood what happened to her after she was released from hospital and was able to look up the translation for appendix in a dictionary. It is not hard to imagine that instead of an appendectomy she may have undergone a hysterectomy without knowing that something much more important than an appendix had been removed. NES physicians might expect their patients to be more autonomous and actively seek information, whereas many NNES patients might still follow communicative strategies they acquired in less patient-centred cultures. As an example of interactions between NES professionals and NNES patients, Suri's case highlights potentially serious implications for patients, who may appear (to the medical professional) to understand more than they actually do, because they do not openly address their knowledge gaps.

Incorrect assumptions and judgments about patients' knowledge of medical terms might also create difficulty in interactions between NNES professionals and their patients. While most patients seemed to be aware of diverging meaning as long as the medical terms were personally relevant to them, their comments also suggested that this fractional awareness did not prevent or safeguard them from misusing medical terms. Nevertheless, the relatively free use of medical terms among patients might lead NNES IMGs to the incorrect assumption that these patients understand and use medical terms correctly (see Section 5.2.2). In turn, physicians might also employ medical terms more liberally in consultations, thus creating the potential for serious miscommunication.

\section{Discussion}

In this exploratory study initial insights have been gathered to illuminate how native language status and medical background knowledge may influence the use, perception and understanding of medical terminology used in cross-linguistic consultations. The interview data was collected with utmost care and participants were frequently reminded that their answers would not be judged in any way in order to avoid them offering only what they believe to be desirable answers. Despite this and other well-known drawbacks such as time-consuming data collection, the 
interview data provided an excellent chance to engage with participants and truly explore their experiences as (Richards 2003). By recruiting patients and medical professionals from NES and NNES backgrounds, the present study takes a first step towards providing a more accurate picture of today's physician-patient interactions, which are increasingly conducted across cultural and linguistic barriers, rather than focusing on monolingual encounters only (see Glickman et al. 2011). It was revealed that NES as well as NNES patients and physicians largely aligned in associating mostly negative emotional responses to the use of medical terminology. In relation to the perception of medical terms, patients and medical professional, regardless of their language background, seemed to judge the number of terms used in consultations very differently. It has previously been suggested that physicians and patients might perceive terms differently because individuals may develop varying degree of sensitivity to technicalness depending on their individual experiences (Bourhis et al. 1989). Through years of practical experience medical professionals may have become so accustomed to using medical terms that they have become desensitised and no longer perceived certain terms as technical. Patients, on the other hand, may lack comparable prolonged medical experience and thus may have been oversensitive and assigned higher technicalness to words frequently used by physicians. The findings in the present study fall in line with those of Bourhis et al. (1989); but most importantly, the research provides the first evidence for differences in the perception of technicalness between individuals with and without a background in medicine.

However, several differences that go beyond the participants' medical background knowledge also surfaced with regard to their attitudes and beliefs towards medical terms as well as their perceptions of technicalness. The interview data revealed that the majority of NNES participants seemed to believe that NES patients know a large number of English medical terms because many (semi-technical) medical terms are frequently used in everyday English discourse, and therefore they often overestimated the level of understanding among NES patients. Moreover, many NNES patients felt they only knew little terminology as they had not been naturally exposed to or instructed in medical terms. Considering that some NNES patients showed great difficulty in understanding the question(s) targeting meaning divergence, it could be argued that their limited English skills may also hinder them from perceiving medical terms as 'technical vocabulary' at all. For these patients, a medical term may just be another unfamiliar English word for which they may seek a first language translation but no additional background information. However, these assumptions should be viewed only within the context of this study and further investigations with larger participant samples are needed to test how different levels of English proficiency influence patients' understanding and perception of medical terms.

In comparison to their respective counterparts, NNES IMGs and patients seemed to be less aware of the potential for meaning divergences and also encountered difficulties in distinguishing between English lay vocabulary items and medical terminology. These findings have important implications for cross-linguistic interactions involving NNES IMGs and for communication skills courses that are targeted at this group. To date most studies on IMGs have focused on their difficulties in relation to Australian slang or lay language (McDonnel1/Usherwood 2008; Pilotto et al. 2007), and some scholars have even suggested that medical language is usually so well known among IMGS that it should not be included in communication training courses (Webber 1995). Furthermore, most medical communication textbooks and courses urge IMGs, or physicians in general, to avoid using medical terms or at least to explain them in an effort to ensure patient comprehension in consultations (see e.g. Silverman et al. 2005; Washer 2009, Australian Medical Council 2007.). However, given that the NNES IMGs in this study had a skewed picture of their NES patients' knowledge capacities and exhibited difficulties to differentiate between lay and medical vocabulary, they might fail to clarify terms used by patients (see Dahm 2011), or fail to provide appropriate explanations of the terms they themselves employ during interactions. In both cases, the threat for miscommunication would be increased. The IMGs in the present study also appeared to differ from NES physicians who generally tend to underestimate their patients' 
knowledge of medical terms (McKinlay 1975; Pratt et al. 1957). While miscalculations in both directions can have far-reaching consequences, it seems likely that the potential for miscommunication also increases significantly in situations where NES patients use medical terms. In these contexts, IMGs, because of their limited local experience, may not realise that their patients could have a different meaning in mind and take the term at face value.

The divergent ideas regarding the perception of terminology and between NES and NNES participants were not only related to their different levels of medical background knowledge, but also to their native language background. In this respect, the interview findings helped to further develop our understanding of technicalness. It has been shown that not only specialised knowledge but also differences in the individuals' language backgrounds can have an impact on the perception of technicalness and by extension on their awareness of meaning divergences. Considering that the concept of technicalness has so far predominantly been used within second language teaching to determine vocabulary for English for specific purposes courses (see Chung 2003), the present study shows that the concept is also a very valuable tool to raise awareness of meaning divergences and to highlight threats for potential miscommunication in specialised discourses, such a legal proceedings or medical encounters.

\section{Conclusion and implications}

In using semi-structured interviews to explore beliefs and attitudes towards the use of medical terminology in multilingual consultations, the findings of this exploratory study suggest that language background as well as medical knowledge influences how individuals perceive medical terminology. Data indicate differences in the way physicians and patients judge the number of terms used in consultations, which confirms the hypothesis that individuals with different levels of medical background knowledge perceive medical terminology differently. NES physicians identified meaning divergence of medical terms as one of the biggest threats to patient-physician communication as patients often misuse or misinterpret terms. In contrast, the majority of other participants only commented on this issue after it was directly raised in a question, and a few NNES patients had significant difficulties understanding the question related to meaning divergences at all. Overall, patients were aware of divergent meanings and potential shifts in the perception of technicalness, but it seems that this awareness only applied to terms that are personally relevant to them. Some IMGs did not immediately grasp the threat posed by meaning divergence and their lack of awareness may be attributed to their non-native language status paired with their limited practical experience in Australia. However, this lack of awareness could lead to IMGs overestimating NES patients' knowledge and understanding of medical terms and as a consequence, IMGs may falsely assume that a medical term means the same to them as to a patient and underestimate the potential for miscommunication.

Guidelines for medical communication commonly call on physicians to avoid or explain medical terminology (e.g. Silverman et al., 2005; Washer 2009). However, considering that in this study patients and physicians were found to perceive different language items as 'real medical terms', these recommendations might not improve consultations at all. Given that physicians seem to have only limited sensitivity to medical terms, physicians might only explain terms they themselves perceive to be truly medical, instead of providing further explanations for terms that are unfamiliar for their patients. Hence, some of the medical terms that patients feel should be explained might remain unexplained in consultations. Physicians could benefit from being made aware of the fact their sensitivity to detect 'medical terms' might shift with increased training and practice, and keeping this knowledge in mind and managing the use of medical terminology accordingly might help to ameliorate interactions with patients.

This study offers a first exploration of the perception of medical terminology and awareness of meaning divergences among participants from various language and medical knowledge backgrounds. Despite the fact that it was not designed to offer a comprehensive account of the impact of medical terminology in consultations, some limitations need to be considered. Different results 
concerning the perception of medical terms and awareness of meaning divergences might have been obtained from a larger participant sample. Future research could, for example, take into account chronic or older patients, whose medical and personal experience might be significantly different to those of the younger and healthier patient participants in this study. In the future, greater insights might be gained by including more locally experienced NNES IMGs to investigate how the number of years of residence and practical experience influence communication difficulties. In general, larger scale studies should be implemented to test whether the findings of this initial examination hold true for a larger and even more diverse participant sample.

Moreover, this study also showed the usefulness of the concept of technicalness in researching perceptions of medical terminology. In addition, the findings add an important new perspective to our theoretical knowledge of technicalness which, as the findings showed, does not only fluctuate with field-specific background knowledge but can also diverge for individuals with different native language backgrounds. Future research, especially within specific discourses, could further explore the concept of technicalness and its applicability in learning and teaching English for specific purposes.

Although exploratory, the findings of this study offer some crucial new insights into the experiences of NNES patients and physicians, and help to highlight that the dangers that are associated with using medical terminology in consultations also threaten communication in cross-linguistic medical encounters. The study takes a first step to increase our understanding of the apparent differences in the perception of medical terms among physicians and patients, and underlines that these variations need to be addressed and further researched in the future in order for patient education or medical communication skills courses to be efficient. With regards to patients, educational programs could utilise personal experience as a foundation to raise patient awareness of meaning divergence. NNES patients could benefit if their physicians highlighted the need to seek additional information by pointing out that the meaning of first language translations may not correspond to the meaning that physicians associate with English medical terms. NNES IMGs should be reminded of the fact that patients might frequently use medical terms without necessarily knowing what they signify. In general, physicians could improve their communication skills if they were consistently reminded, for example in communication or professional development programs, that medical experience may alter their perception of medical terminology and render their efforts to avoid or explain medical terms fruitless.

\section{References}

Allotey, Pascale A/Reidpath, Daniel D 1999: Multicultural issues in general practice. In Current Therapeutics 40, 3537.

Australian Bureau of Statistics 2008: Country of birth of person by proficiency in spoken English/language by sex (No. 2068.0 - 2006 Census Tables). Canberra, Australian Capital Territory: Author. Retrieved 31 August 2011 from AusStats database.

Australian Bureau of Statistics 2011: 2009-2010 Migration Australia (No. 3142.0). Canberra, Australian Capital Territory: Author. Retrieved 31 August 2011 from AusStats database.

Australian Medical Council 2007: AMC handbook of clinical assessment: Annotated multidisciplinary clinical tasks. Canberra: Australian Medical Council.

Bates, Joanna/Andrew, Rodney 2001: Untangling the roots of some IMGs' poor academic performance. In Academic Medicine 76(1), 43-46.

Birrell, Bob/Schwartz, Andrew 2006: Accreditation of overseas trained doctors: The continuing crisis. In People and Place 14, 37-47.

Bourhis, Richard Y/Roth, Sharon/MacQueen, Glenda 1989: Communication in the hospital setting: a survey of the medical and everyday language use amongst patients, nurses and doctors. In Social Science and Medicine 28, 339346.

Chung, Teresa Mihwa 2003: A corpus comparison approach for terminology extraction. In Terminology 9, 221-246.

Cooke, M W/Wilson, S/Cox, P/Roalfe, A 2000: Public understanding of medical terminology: non-English speakers may not receive optimal care. In Journal of Accident and Emergency Medicine 17, 119-121. 
Dahm, Maria R 2011: Exploring perception and use of everyday language and medical terminology among international medical graduates in a medical ESP course in Australia. In English for Specific Purposes 30, 186-197.

Dahm, Maria R 2012: Tales of time, terms and patient information-seeking behaviour - an exploratory qualitative study. In Health Communication 27, 682-689.

Fields, Aaron M/Freiberg, Craig S/Fickenscher, Alexandra/Shelley, Kirk H 2008: Patients and jargon: are we speaking the same language? In Journal of Clinical Anesthesia 20, 343-346.

Frank, Ruth A 2000: Medical communication: non-native English speaking patients and native English speaking professionals. In English for Specific Purposes 19, 31-62.

Gittelman, Michael A/Mahabee-Gittens, Melinda/Gonzalez-del-Rey, Javier 2004: Common medical terms defined by parents: are we speaking the same language? In Pediatric Emergency Care 20, 754-758.

Glickman, Seth W./Ndubuizu, Adanma/Weinfurt, Kevin P./Hamilton, Carol D./Glickman, Lawrence T./Schulman, Kevin A./Cairns, Charles B. 2011: The case for research justice: Inclusion of patients with limited English proficiency in clinical research. In Academic Medicine 86, 389-393.

Groves, Michele/Fitzgerald, Jennifer (ed.) 2010: Communication Skills in Medicine. Promoting Patient-Centred Care. Melbourne: IP Communications.

Hadlow, Jan/Pitts, Marian 1991: The understanding of common health terms by doctors, nurses and patients. In Social Science and Medicine 32, 193-196.

Heigham, Juanita/Croker, Robert A (ed.) 2009: Qualitative Research in applied linguistics. A practical introduction. Basingstoke, Hampshire; New York, NY: Palgrave Macmillan.

Helman, Cecil G 2007: Culture, Health and Illness. London: Hodder Arnold.

Koch-Weser, Susan/Dejong, William/Rudd, Rima E 2009: Medical word use in clinical encounters. In Health Expectations 12, 371-382.

Knox, Stephanie. A./Britt, Helena. 2002: A comparison of general practice encounters with patients from Englishspeaking and non-English speaking backgrounds. In Medical Journal of Australia 177, 98-101.

Levin, M E 2006: Different use of medical terminology and culture-specific models of disease affecting communication between Xhosaspeaking patients and English-speaking doctors at a South African paediatric teaching hospital. In South Africal Medical Journal 96, 1080-1084.

McDonnell, L./Usherwood, T. 2008: International medical graduates: Challenges faced in the Australian training program. In Australian Family Physician 37, 481-484.

McKinlay, John B 1975: Who is really ignorant - physician or patient? In Journal of Health and Social Behavior 16, 3-11.

Murray, S./Buller, A. M. 2007: Exclusion on grounds of language ability - A reporting gap in health services research? In Journal of Health Services Research and Policy 12, 205-208.

Nation, I.S.P. 2001: Learning vocabulary in another language. Cambridge: Cambridge University Press.

Nation, I.S.P. 1990: Teaching and Learning Vocabulary. New York: Newbury House.

Pauwels, A 1994: Applying linguistic insight in intercultural communication to professional training programs: an Australian case study. In Multilingua 13, 195-212.

Perez, Miguel A/Luquis, Raffy R (eds.) 2008: Cultural Competence in Health Education and Health Promotion. San Francisco: Jossey-Bass.

Pilotto, Louis S/Duncan, Geraldine F/Anderson-Wurf, Jane 2007: Issues for clinicians training international medical graduates: a systematic review. In Medical Journal of Australia 187, 225-228.

Pratt, Lois/Seligmann, Arthur /Reader, George 1957: Physicians' views on the level of medical information among patients. In American Journal of Public Health 47, 1277-1283.

QSR International Pty Ltd 2008: NVivo qualitative data analysis software.

Read, John 2004: Plumbing the depths: How should the construct of vocabulary knowledge be defined? In Bogaards P./Laufer B (eds.), Vocabulary in a second language. Selection, acquisition, and testing. Amsterdam, Philadelphia, PA: John Benjamins.

Richards, K 2003: Qualitative inquiry in TESOL. Houndsmill, Basingstoke: Plagrave.

Roberts, Celia/Moss, Becky/Wass, Val/Sarangi, Srikant/Jones, Roger 2005: Misunderstandings: a qualitative study of primary care consultations in multilingual settings, and educational implications In Medical Education 39, 465-475.

Schmitt, Norbert 2000: Vocabulary in language teaching. Cambridge: Cambridge University Press.

Schoonen, Rob/Verhallen, Marianne 2008:. The assessment of deep word knowledge in young first and second language learners. In Language Testing 25, 211-236. 
Schouten, Barbara C./Meeuwesen, Ludwien 2006: Cultural differences in medical communication: A review of the literature In Patient Education and Counseling 64, 21-34.

Silverman, Jonathan/Kurtz, Suzanne/Draper, Juliet 2005: Skills for communicating with patients. Oxon: Radcliffe.

Strauss, Anselm/Corbin, Juliet 2008: Basics of qualitative research. Techniques and procedures for developing grounded theory. Thousand Oaks, Ca; London: Sage Publications.

Street, Richard L., Jr. 2003: Communication in medical encounters: an ecological perspective. In Thompson, Teresa L./ Dorsey, Alicia M./Miller, Katherine I./Parrott, Roxanne (eds.), Communication in medical encounters: an ecological perspective. Mahwah, New Jersey: Lawrence Erlbaum.

Tannen, Deborah/Wallat, Cynthia 1987: Interactive frames and knowledge schemas in interaction: examples from a medical examination/interview. In Social Psychology Quarterly 50, 205-216.

Washer, Peter (ed.) 2009: Clinical Communication Skills. Oxford: Oxford University Press.

Webber, Pauline 1995: Speaking practice in the medical English classroom. In IRAL 33, 64-70. 


\section{Appendix - Interview schedules}

\section{Patients}

1. Did you ever experience difficulty in communicating with either your "regular" doctor or another GP or specialist you have seen? If yes, could you please describe these problems in more detail and also tell me how they were resolved?

2. Did you ever have communication problems caused by the use of medical terms? Did the doctor provide any explanations for the medical terms?

3. Did you ask anyone else to explain medical terms to you after you saw the doctor?

4. Have you ever looked up the meaning of a medical term in a dictionary or on the Internet?

5. Do you think you have adequate vocabulary to tell your doctor exactly what is wrong with you?

5.1. NNS: Do you think you only have problems understanding medical terms in the Australian context because English is not your native language? Do you think native speakers of English (NSE) have similar problems?

5.2. NES: Do you have problems understanding medical terminology? Do you think nonnative speakers have greater problems understanding medical terms than native speakers?

6. Think of a medical condition of a personal or non-personal nature that you feel comfortable discussing.

6.1. What words do you use to describe your symptoms or affected body parts areas?

6.2. What words do you use to talk about the "technical aspects" of this condition, e.g. treatment, medication etc.

7. When you use a medical term, do you think it means the same to your doctor as it does to you?

8. Regarding patient-doctor communication, what do you judge the amount of terms used, and what do you think in general about the use of medical terminology by doctor?

9. What kind of language do you prefer your doctor to use and why?

10. Do you think better of a doctor that uses more plain English or more technical terms?

10.1. Judging specific characteristics of doctors in connection to the terms they use: Do you associate professional medical expertise to the use of lay or of technical terminology? Do you associate empathy with patients to the use of lay or of technical terminology?

11. Can you think of a word or words that you have come across in everyday life that you would consider to me a medical term?

\section{Physicians}

1. Did you ever experience difficulty in communicating with either your patients? If yes, could you please describe these problems in more detail and also tell me how they were resolved?

2. Did you ever have communicative problems caused by the use of medical terms? Do you generally provide explanations for the terms you use?

3. Do you think that your patients have adequate vocabulary to describe their symptoms and follow your questions/explanations/instructions?

How do you make sure your patients have understood your questions/explanation/ instructions?

4. Do you think patients have difficulty understanding medical terms regardless of their native language? 
5. Do you adjust your use of medical terms according to the language background of your patients?

6. What factors other than (native) language do you think contribute to problems in understanding medical terminology?

7. When you use a medical term do you think it means the same to your patient as it does to you?

7.1. Are there any terms you avoid because they cause too much confusion?

7.2. Are there any terms you thought were generally known, but which you still have to explain to most patients?

7.3. Are there any terms patients frequently use in a wrong way? How so and do you think your patients are aware of their misuse? How do you try to prevent/resolve miscommunication/misunderstanding caused by differences in understanding?

8. Do you encourage patients to seek more information independently or do you hand out leaflet or information brochures?

How do you feel about "informed" patients that use a lot of medical terms?

9. Regarding patient-doctor communication, how do you judge the amount of terms used, and what do you think in general about the use of medical terminology by doctors?

10. What kind of language do you prefer to use and why? How do you generally feel about the amount of medical terms you use in your consultations?

11. From a patient's perspective: What factors would a "good" doctor take into account when deciding what terminology to use?

11.1 Judging specific characteristics of doctors in connection to the terms they use Do patients associate professional medical expertise to the use of lay or of technical terminology?

Do patients associate empathy with patients to the use of lay or of technical terminology?

\section{Additional questions for (or regarding) NNES IMGs}

12. In general, what do you think is/will be most problematic for you/NNES IMGs in consultations?

13. Regarding English proficiency what do you think causes/will cause the most difficulties for you/NNES IMGs and why: lay vocabulary, idioms, medical slang (acronyms) or medical terminology?

14. What differences are there between the use of medical terms by patients and doctors in your home country and in Australia?

15. Would you use your native language in consultations if it would help communicating with patients? 